\title{
Dynamic Contrast-Enhanced and Intravoxel Incoherent Motion MRI Biomarkers Are Correlated to Survival Outcome in Advanced Hepatocellular Carcinoma
}

\author{
Bang-Bin Chen ${ }^{1,2}{ }^{\mathbb{D}}$, Yu-Yun Shao ${ }^{3,4,5} \mathbb{D}$, Zhong-Zhe Lin ${ }^{3,5}$, Chih-Hung Hsu ${ }^{3,4,5}$, Ann-Lii Cheng $3,4,5,6$, \\ Chiun Hsu ${ }^{3,4,5}$, Po-Chin Liang ${ }^{1,2,7}$ and Tiffany Ting-Fang Shih 1,2,* \\ 1 Department of Medical Imaging, National Taiwan University Hospital, Taipei 100, Taiwan; \\ bangbin@gmail.com (B.-B.C.); pochin.liang@gmail.com (P.-C.L.) \\ 2 Department of Radiology, College of Medicine, National Taiwan University, Taipei 100, Taiwan \\ 3 Department of Oncology, National Taiwan University Hospital, Taipei 100, Taiwan; \\ yuyunshao@gmail.com (Y.-Y.S.); zzlin7460@ntu.edu.tw (Z.-Z.L.); chihhunghsu@ntu.edu.tw (C.-H.H.); \\ alcheng@ntu.edu.tw (A.-L.C.); hsuchiun@ntu.edu.tw (C.H.) \\ 4 Graduate Institute of Oncology, College of Medicine, National Taiwan University, Taipei 100, Taiwan \\ 5 Department of Medical Oncology, National Taiwan University Cancer Center, Taipei 100, Taiwan \\ 6 Department of Internal Medicine, National Taiwan University Hospital, Taipei 100, Taiwan \\ 7 Department of Medical Imaging, National Taiwan University Hospital Hsin-Chu Branch, \\ Hsin-Chu City 300, Taiwan \\ check for \\ * Correspondence: ttfshih@ntu.edu.tw; Tel.: + 886-2-2312-3456 (ext. 65568); Fax: +886-2-2322-4552
}

updates

Citation: Chen, B.-B.; Shao, Y.-Y.; Lin, Z.-Z.; Hsu, C.-H.; Cheng, A.-L.; Hsu, C.; Liang, P.-C.; Shih, T.T.-F. Dynamic Contrast-Enhanced and Intravoxel Incoherent Motion MRI Biomarkers Are Correlated to Survival Outcome in Advanced Hepatocellular Carcinoma. Diagnostics 2021, 11, 1340. https://doi.org/10.3390/

diagnostics11081340

Academic Editors: Thomas Geyer and Saif Afat

Received: 8 July 2021

Accepted: 23 July 2021

Published: 26 July 2021

Publisher's Note: MDPI stays neutral with regard to jurisdictional claims in published maps and institutional affiliations.

Copyright: (c) 2021 by the authors. Licensee MDPI, Basel, Switzerland. This article is an open access article distributed under the terms and conditions of the Creative Commons Attribution (CC BY) license (https:// creativecommons.org/licenses/by/ $4.0 /)$.
Abstract: Objective: This study assessed dynamic contrast-enhanced (DCE)-MRI and intravoxel incoherent motion diffusion-weighted imaging (IVIM DWI) parameters to prospectively predict survival outcomes in participants with advanced hepatocellular carcinoma (HCC) who received lenalidomide, a dual antiangiogenic and immunomodulatory agent, as second-line therapy in a Phase II clinical trial. Materials and methods: Forty-four participants with advanced HCC who had progression after sorafenib as first-line treatment were prospectively enrolled. Pretreatment MRI parameters-obtained from DCE-MRI (peak, slope, AUC, $K^{\text {trans }}, K_{e p}$, and $V_{e}$ ), apparent diffusion coefficient (ADC), and IVIM DWI (pure diffusion coefficient (D), pseudodiffusion coefficient $\left(\mathrm{D}^{*}\right)$, and perfusion fraction (f)) - were derived from the largest hepatic tumor. The Cox model was used to investigate the associations of the parameters with progression-free survival (PFS) and overall survival (OS). Results: Median PFS and OS were 2.3 and 8.0 months, respectively. Univariate analysis showed that participants with a high slope $(p=0.024), \mathrm{K}_{\mathrm{ep}}(p<0.001)$, and $\operatorname{ADC}(p=0.018)$ values had longer PFS than those with low values; participants with a small tumor size $(p=0.006)$, high slope $(p=0.01)$, ADC $(p=0.015)$, and $\mathrm{f}(p=0.012)$ values had longer OS than those with low values did. Cox multivariable analysis revealed that $\mathrm{K}_{\mathrm{ep}}(p<0.001)$ and $\operatorname{ADC}(p=0.009)$ remained independent predictors of PFS; slope $(p=0.003)$ and ADC $(p=0.009)$ remained independent predictors of OS. Moreover, $\mathrm{K}_{\mathrm{ep}}$ and slope were still significant after Bonferroni correction was performed $(p<0.005)$. Conclusion: Both pretreatment DCE-MRI and IVIM DWI parameters, especially slope and ADC, may predict PFS and OS in participants with HCC receiving lenalidomide as second-line therapy.

Keywords: magnetic resonance angiography; diffusion MRI; hepatocellular carcinoma; survival; lenalidomide

\section{Introduction}

Hepatocellular carcinoma (HCC) is the third highest cause of cancer-related death, with an increasing global incidence. For advanced HCC, sorafenib is recommended as a first-line treatment according to the Barcelona Clinic Liver Cancer staging system [1]; however, its response rate is low, with a complete response rate of $0 \%$ and a partial response rate of only $2.2 \%$ in two pivotal Phase III trials [2-4]. Three tyrosine kinase inhibitors, namely, 
regorafenib, cabozantinib, and ramucirumab, and two checkpoint inhibitors, namely, nivolumab and pembrolizumab, were approved by the US Food and Drug Administration as second-line treatment options after prior sorafenib treatment [5]. Lenalidomide, which has both antiangiogenic and immunomodulatory effects, also demonstrated efficacy as a second-line treatment for advanced HCC [6,7]. Patients with advanced HCC generally have poor survival outcomes; therefore, before the administration of treatments, determining image biomarkers that can be used to identify patients who are likely to benefit from such treatments is imperative.

Dynamic contrast-enhanced (DCE) MRI is used to measure tissue perfusion, blood flow, and vascularity by analyzing a tissue signal-enhancement curve after contrast-agent administration [8]. It can be used to measure changes in tumor vascular permeability induced by antiangiogenic agents. In several clinical trials of new targeted therapies for HCC, DCE-MRI biomarkers using conventional gadolinium-based contrast agents have been used as early surrogates to predict clinical response and survival outcome [7,9-11]. For example, in patients who had received sorafenib plus metronomic tegafur/uracil therapy, the forward volume transfer constant $\left(\mathrm{K}^{\text {trans }}\right)$ correlated well with tumor response and survival [10]. Another study found that high peak (difference between maximal and baseline signal intensity) reduction within one week was a favorable prognostic factor after systemic treatment [8]. However, vascular response determined by $>40 \% \mathrm{~K}^{\text {trans }}$ reduction at 2 weeks did not correlate with treatment response after lenalidomide [7] and vandetanib [10] treatments.

Diffusion-weighted imaging (DWI), in which changes in the cellular density of tissue can be estimated on the basis of an apparent diffusion coefficient (ADC), showed considerable promise as an imaging biomarker in HCC $[12,13]$. The intravoxel incoherent motion (IVIM) model using multiple $b$ values can be used to derive pseudodiffusion $\left(\mathrm{D}^{*}\right)$, pure diffusion characteristics (D), and perfusion fraction (f). Studies showed that IVIM imaging biomarkers may differentiate histological grades of HCC $[14,15]$ and predict prognosis in transarterial chemoembolization treatment [16]. For example, ADC and IVIM-derived $D$ values exhibited high diagnostic performance in differentiating high-grade HCC from low-grade HCC [14]. ADC and Dslow ratios calculated at 24-48 h relative to baseline were reported to be independent predictors of response for HCC after transarterial chemoembolization [17]. However, according to our review of the literature, data concerning the combined use of DCE-MRI and IVIM DWI for predicting survival outcomes in patients with HCC are limited. Currently, no imaging biomarker is available to predict survival outcome in patients receiving second-line targeted therapy after first-line sorafenib treatment.

To address the aforementioned literature gap, we conducted this study with the purpose of assessing DCE-MRI and IVIM DWI biomarkers to prospectively predict survival outcomes in participants with advanced HCC who had progression after first-line sorafenib and received lenalidomide as second-line therapy. We hypothesized that pretreatment MRI biomarkers obtained from DCE-MRI and IVIM DWI would predict the survival outcome before lenalidomide treatment.

\section{Materials and Methods}

This prospective open-label, single-arm, single-center, investigator-initiated Phase II clinical trial, was approved by the Institutional Research Ethics Committee (protocol code: NTUH-REC No. 201105063MB, date of approval: 2 August 2011) of our institute (www.clinicaltrials.gov NCT01545804, Last accessed on 10 July 2021) [7]. Written informed consent was obtained from all the participants. No financial support from the industry was received. The authors had full control of the data and submitted information.

\subsection{Study Participants}

The inclusion criteria were as follows: receiving a histological or clinical diagnosis of HCC, having documented progression under treatment with or intolerance to sorafenib or other systemic therapy, having an Eastern Cooperative Oncology Group score of 0 or 
1, being classified into Child-Pugh class A, and having at least one measurable lesion according to Response Evaluation Criteria in Solid Tumors (RECIST) 1.1 [18]. The exclusion criteria are listed in Figure 1. Participants received lenalidomide ( $25 \mathrm{mg} /$ day orally) on days 1-21 every 4 weeks. Tumor response was assessed according to RECIST 1.1 after 4 and 8 weeks of treatment and every 8 weeks thereafter. Participants were followed until death. The primary endpoint was 6-month progression-free survival (PFS) using RECIST 1.1. We collected clinical data, namely, age, sex, hepatitis status, cirrhosis, extrahepatic metastasis, macroscopic vascular invasion, alpha-fetoprotein, treatment received, and mortality date.

94 patients with advanced HCC who failed first-line systemic therapy screened

Inclusion:

1. Histological or clinical diagnosis of HCC

Exclusion:

2. Documented progression on or intolerance to first-line systemic therapy

3. ECOG performance status 0 or 1

4. Child-Pugh classification $A$

5. At least one measurable lesion according to RECIST 1.1

1. Patient refuse $(n=11)$

2. High liver transaminase level $(n=9)$

3. Prior tumor rupture $(n=3)$

4. Leukopenia $(n=3)$

5. No measurable lesions $(n=2)$

6. Recent gastrointestinal bleeding $(n=2)$

7. $>1$ line of systemic therapy $(n=2)$

8. Unconfirmed diagnosis $(n=2)$

9. Others $(n=5)$

\begin{tabular}{|l}
\hline 55 patients enrolled \\
All eligible for response and safety evaluation and survival follow-up \\
$\qquad \begin{array}{l}\text { Exclusion: } \\
\text { 1. No viable tumor in the liver }(n=8) \\
\text { 2. Early treatment discontinuation }(n=2) \\
\text { 3. Unavailable facility }(n=1)\end{array}$ \\
\begin{tabular}{|l} 
Eligible for DCE-MRI and IVIM-MRI $(n=44)$ \\
\hline
\end{tabular}
\end{tabular}

Figure 1. Summary of inclusion and exclusion criteria, and final study population. HCC = hepatocellular carcinoma; ECOG = Eastern Cooperative Oncology Group; RECIST = response evaluation criteria in solid tumors; DCE = dynamic contrast-enhanced; IVIM = intravoxel incoherent motion.

We initially enrolled 55 participants during the period from June 2012 to June 2014. Lastly, we included 44 participants (men: 39; women: 5; median age: $60.1 \pm 11.6$ years; range 31-80 years) who had undergone a pretreatment MRI examination in this study (Figure 1). Data for all 44 participants were reported in our previous study [7], which focused on the correlation of a single DCE-MRI parameter $\left(\mathrm{K}^{\text {trans }}\right)$ with treatment outcomes. In our previous study, the vascular response determined by a $>40 \%$ decline in $\mathrm{K}^{\text {trans }}$ was not associated with any treatment outcome [7]. In contrast, in the present study, we investigated the correlation of pretreatment DCE-MRI and IVIM biomarkers with PFS and overall survival (OS), which had not been previously reported.

\subsection{MRI Protocol}

Each participant received liver MRI at $3 \mathrm{~T}$ (Magnetom Verio; Siemens Healthcare, Erlangen, Germany) through a 32-channel phased-array coil. Routine MRI sequences included the following: a half-Fourier single-shot turbo spin-echo sequence, a breath-hold T1-weighted dual-echo (inphase and opposed-phase) volumetric interpolated breath-hold examination sequence, and a T2-weighted fast spin-echo sequence with fat suppression. Three gradient directions were chosen for DWI (Table 1). 
Table 1. MRI parameters.

\begin{tabular}{|c|c|c|c|c|c|c|c|c|}
\hline Sequence & $\begin{array}{c}\text { TR } \\
\text { (msec) }\end{array}$ & $\begin{array}{c}\mathrm{TE} \\
\text { (msec) }\end{array}$ & $\begin{array}{l}\text { Flip Angle } \\
\text { (degrees) }\end{array}$ & Matrix & $\begin{array}{c}\text { Field of } \\
\text { View (mm) }\end{array}$ & $\begin{array}{l}\text { Slice Thick- } \\
\text { ness/Gap } \\
(\mathrm{mm})\end{array}$ & NEX & $\begin{array}{l}\text { Acquisition } \\
\text { Time (min) }\end{array}$ \\
\hline Coronal HASTE & 1400 & 93 & 160 & $640 \times 640$ & $320 \times 320$ & $5 / 0$ & 1 & $0: 59$ \\
\hline $\begin{array}{l}\text { Axial T1WI VIBE (in- } \\
\text { and opposed-phase) }\end{array}$ & 4.2 & 2.5 & 10 & $512 \times 416$ & $350 \times 284$ & $3 / 0$ & 1 & $0: 52$ \\
\hline Axial T2WI FS & 2610 & 96 & 123 & $640 \times 440$ & $340 \times 234$ & $6 / 0$ & 1 & 1:04 \\
\hline DWI $(b=50,500,1000)$ & 7300 & 83 & 90 & $384 \times 300$ & $4000 \times 313$ & $6 / 0$ & 2 & 4:08 \\
\hline $\begin{array}{c}\operatorname{IVIM}(b=0,10,20,30 \\
40,50,60,70,80,90,100 \\
200,300,400,500,1000)\end{array}$ & 4438 & 77.2 & 180 & $182 \times 150$ & $385 \times 317$ & $6 / 0$ & 2 & 14:06 \\
\hline DCE-MRI & 4.2 & 2.5 & 9 & $420 \times 448$ & $400 \times 313$ & $5 / 0$ & 1 & $\begin{array}{c}2: 50 \\
\text { (25 sets) }\end{array}$ \\
\hline Axial T1WI postcontrast & 141 & 2.5 & 70 & $320 \times 220$ & $340 \times 234$ & $6 / 0$ & 1 & $1: 00$ \\
\hline
\end{tabular}

Note: HASTE = half-Fourier single-shot turbo spin-echo; T1WI = T1-weighted imaging; VIBE = volumetric interpolated breath-hold examination; T2WI FS = T2-weighted imaging with fat suppression; DWI = diffusion-weighted imaging; IVIM = intravoxel incoherent motion; $\mathrm{DCE}=$ dynamic contrast-enhanced; $\mathrm{TR}=$ repetition time; $\mathrm{TE}=$ echo time; $\mathrm{NEX}=$ number of averages.

Axial liver IVIM imaging was performed using a free-breathing single-shot echoplanar imaging protocol in which diffusion gradients were applied in three orthogonal directions [19]. Encoding was performed using $16 b$ values $(0,10,20,30,40,50,60,70,80$, $90,100,200,300,400,500$, and $1000 \mathrm{~s} / \mathrm{mm}^{2}$ ) before injection of gadolinium chelate.

DCE-MRI was performed on 24 consecutive oblique coronal sections using a threedimensional T1-weighted volumetric interpolated breath-hold examination (VIBE) sequence. Gadobutrol at a dose of $0.1 \mathrm{mmol} / \mathrm{kg}$ (Gd-DO3A-butrol, Gadovist; Bayer Pharma, Leverkusen, Germany) was injected at a rate of $2 \mathrm{~mL} / \mathrm{s}$ into an antecubital vein by using an automated injector, followed by a $20 \mathrm{~mL}$ saline flush. All the participants were instructed to hold their breath for as long as they could tolerate, and then breathe slowly and smoothly during imaging. The total acquisition time for DCE-MRI was $2 \mathrm{~min}$ and $50 \mathrm{~s}$, with a temporal resolution of $6.4 \mathrm{~s}$; moreover, 600 dynamic images were obtained for each participant. Lastly, static fat-suppressed axial contrast-enhanced T1-weighted imaging was performed to image the whole liver. All imaging procedures were performed by the same technician.

\subsection{Image Analysis}

\subsubsection{IVIM Modeling of DWI}

DWI data were coregistered using an image-based nonaffine registration algorithm (dynamic field correction), with a $b$ value of $0 \mathrm{~s} / \mathrm{mm}^{2}$ serving as a reference. For calculating the standard $\mathrm{ADC}$ value, monoexponential fitting of the data was calculated by a least-squares fit equation $(\mathrm{S} / \mathrm{S} 0=\exp (-\mathrm{b} \times \mathrm{ADC}))$ using all $\mathrm{b}$ values, where $\mathrm{S} 0$ is the signal without diffusion gradient, and $S$ is the signal with a diffusion weighting $[20,21]$. Subsequently, IVIM parameters were derived with all $b$ values serving as input data on a voxel-by-voxel basis. The following formula was used to derive the IVIM parameters [22]:

$$
\mathrm{S}(b) / \mathrm{S}(0)=\mathrm{f} \times \exp \left[-b\left(\mathrm{D}^{*}\right)\right]+(1-\mathrm{f}) \times \exp [-b(\mathrm{D})
$$

where $D$ denotes a pure diffusion coefficient; $D^{*}$ denotes a pseudodiffusion coefficient; $f$ denotes a perfusion fraction; and $S(b)$ and $S(0)$ denote signal intensity with and without the application of the diffusion gradient, respectively.

D values were estimated from signal-intensity data at high $b$ values $\left(b>200 \mathrm{~s} / \mathrm{mm}^{2}\right)$.

Regions of interest (ROIs) were drawn using commercial Osirix ${ }^{\circledR}$ medical-image software [23]. ADC and IVIM values were calculated using an Osirix plugin (ADCmap, version 2.4). For nonlinear least-squares fitting, the Levenberg-Marquardt algorithm was implemented in the plugin.

All image measurements were performed by a radiologist (B.B.C) with 12 years of experience in liver MRI interpretation; the radiologist was blinded to the clinical history 
of the participants. A single representative ROI was manually traced along the margin of the tumor on ADC maps on the section showing the largest tumor cross-sectional area. Subsequently, the ROI was copied and pasted to the images of IVIM parameters. The ROI location was visually checked to prevent misregistration due to motion. The mean (range) ROI area was $38.4 \pm 44.4(1.8-174.5) \mathrm{cm}^{2}$ (Figures 2-4).

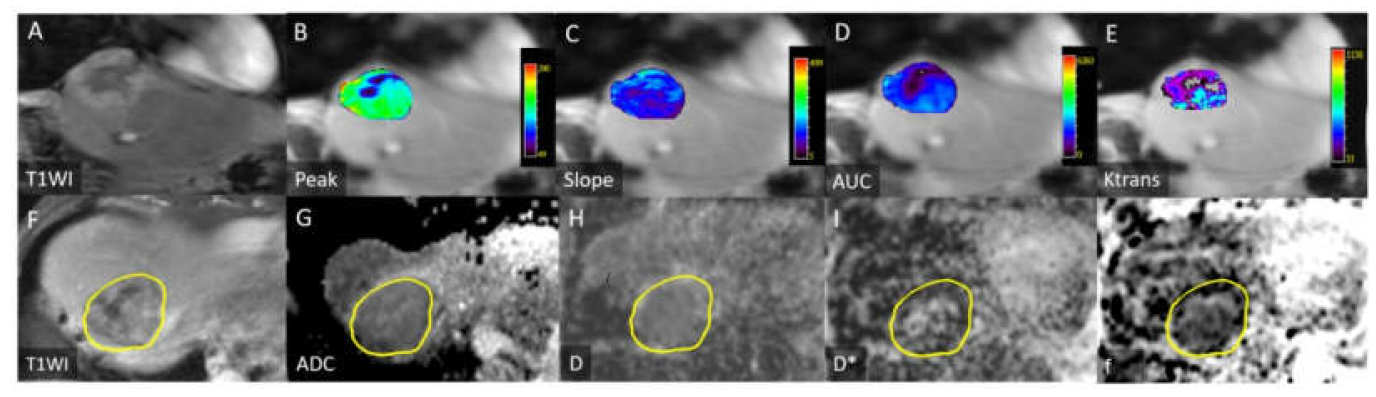

Figure 2. Images for a 77-year-old man with progression-free survival of 3.6 months and overall survival of 10 months. (A) Coronal contrast-enhanced T1-weighted image depicting a hepatocellular carcinoma in the right liver dome. Peak (B), slope (C), AUC (D), and $\mathrm{K}^{\text {trans }}(\mathrm{E})$ values were $15.4(\%)$, $12.8(1 / \mathrm{s}), 1066(/ 10 \mathrm{~s})$, and $0.079\left(\mathrm{~min}^{-1}\right)$, respectively. (F) Axial contrast-enhanced T1-weighted images depicting heterogeneous enhancement of the tumor. ADC $(\mathbf{G}), \mathrm{D}(\mathbf{H}), \mathrm{D}^{*}(\mathbf{I})$, and $\mathrm{f}(\mathbf{J})$ values were $1.38 \times 10^{-3}, 1.16 \times 10^{-3}$, and $100 \times 10^{-3} \mathrm{~s} / \mathrm{mm}^{2}$, and $11.7 \%$, respectively. Yellow circles in Figure $2 \mathrm{~F}-\mathrm{J}$ mark tumor margin.

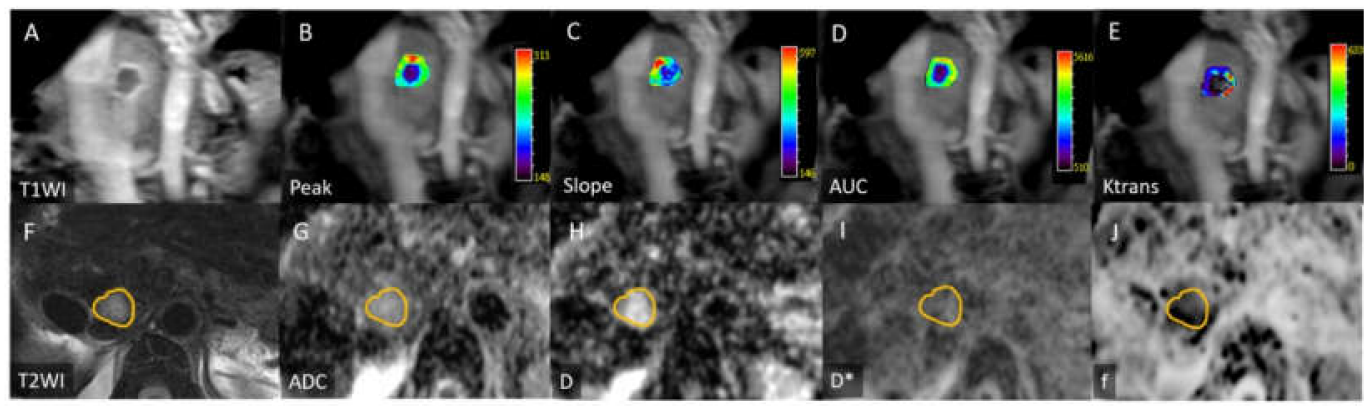

Figure 3. Images for a 71-year-old woman with progression-free survival of 5.5 months and overall survival of 29.8 months. (A) Coronal contrast-enhanced T1-weighted image depicting a hepatocellular carcinoma with peripheral enhancement in the right liver dome. Peak (B), slope (C), AUC (D), and $\mathrm{K}^{\text {trans }}(\mathrm{E})$ values were $36.2(\%), 38.4(1 / \mathrm{s}), 3552(/ 10 \mathrm{~s})$, and $0.119\left(\mathrm{~min}^{-1}\right)$, respectively. (F) Axial T2-weighted image depicting mild hyperintense signal intensity of the tumor. ADC $(\mathbf{G}), \mathrm{D}(\mathbf{H}), \mathrm{D}^{*}(\mathbf{I})$, and $f(J)$ values were $1.96 \times 10^{-3}, 1.6 \times 10^{-3}$, and $100 \times 10^{-3} \mathrm{~s} / \mathrm{mm}^{2}$, and $16.7(\%)$, respectively. Yellow circles in Figure 3F-J mark tumor margin.

\subsubsection{DCE-MRI}

DCE-MRI data were analyzed using a commercial software tool (MIStars; Apollo Medical Imaging, Melbourne, Australia), and motion correction was performed. The motion-correction algorithm used a 2D rigid body with three adjustable parameters: translation in $\mathrm{x}$ and $\mathrm{y}$, and inplane rotation. The following semiquantitative parameters were obtained by analyzing the characteristics of tumor enhancement curves: Peak (maximal signal intensity minus baseline signal intensity), slope (maximal ascending slope of the curve), and initial area under the gadolinium concentration-time curve (AUC) at $60 \mathrm{~s}$ after contrast injection. Furthermore, pharmacokinetic modeling was calculated using a single-input two-compartment model with the aorta as arterial input function $[9,24]$. Three quantitative parameters ( $\mathrm{K}^{\text {trans }}$ : forward volume transfer constant, $\mathrm{K}_{\mathrm{ep}}$ : reverse rate transfer constant, and $\mathrm{V}_{\mathrm{e}}$ : extravascular extracellular space volume per unit volume of tissue) were automatically calculated pixel by pixel using a constrained nonlinear least-squares 
fitting algorithm with adjustable delay time. All ROIs were drawn by the same radiologist (B.B.C). The necrotic area within a tumor was included. The mean (range) ROI in the tumor was $49.5 \pm 59.3(2.1-288) \mathrm{cm}^{2}$ (Figures 2 and 3).

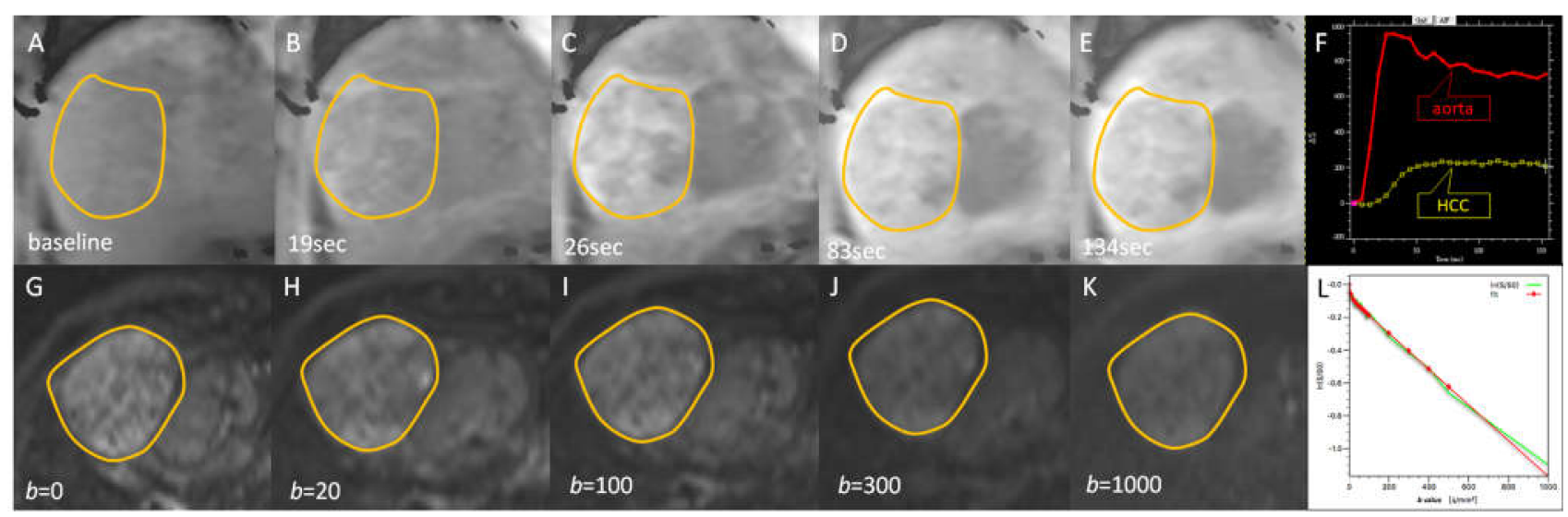

Figure 4. Representative signal dynamics images for DCE-MRI and DWI/IVIM in a 53-year-old man. (A-E) Coronal contrast-enhanced T1-weighted image depicting dynamic enhancement of a hepatocellular carcinoma (HCC) in right liver dome. (F) DCE-MRI enhancement curves of aorta (red) and HCC (yellow). (G-K) Axial DWI/IVIM images with different b values. (L) Fitting diffusion curve in whole HCC lesion. Yellow circles in Figure 4F-J,G-K mark tumor margin.

In addition, to evaluate the interobserver variability of these parameters, ROI placement was performed for all MR imaging examinations in all patients by another radiologist (T.T.F.S., with 27 years of experience in MR imaging).

\subsection{Statistical Analysis}

Interobserver variability was calculated by using an intraclass correlation coefficient. Spearman's correlation (rho) analysis was used to determine the correlation between MRI parameters (very weak correlation: <0.2; weak: 0.20-0.39; moderate: 0.40-0.59; strong: 0.60-0.79; very strong: 0.80-1.0). PFS and OS were measured from the date of examination to the date of progression and to the date of death, respectively. MRI parameters derived for participants with short and long OS (determined by a median OS period of 8.0 months) were compared using the nonparametric Mann-Whitney U test. Survival was analyzed using the Mantel-Cox log-rank test and was presented as Kaplan-Meier survival curves. For MRI parameters, the optimal cutoff for the predictor was estimated by using the maximally selected rank statistics (maxstat package) in R statistical software ( $R$, version 4.0.3; R Foundation for Statistical Computing, Vienna, Austria). Age, sex, tumor size, alpha-fetoprotein, Eastern Cooperative Oncology Group performance status, Child-Pugh score, cirrhosis, macroscopic vascular invasion, extrahepatic spread, and MRI parameters were included in univariate Cox proportional-hazards regression models for PFS and OS. Variables with $p$ values of $<0.05$ in the univariate analysis were used as inputs for a multivariable model. All the statistical analyses were performed using the $\mathrm{R}$ software and SPSS for Windows 22 (SPSS, Chicago, IL, USA). $p<0.05$ was considered to indicate a significant difference. Bonferroni correction was applied to adjust for multiple comparisons $(p<0.005)$.

\section{Results}

\subsection{Participants' Characteristics}

Mean tumor size was $38.4 \pm 44.4 \mathrm{~cm}^{2}$ (range, $1.8-174.5 \mathrm{~cm}^{2}$; median, $17.5 \mathrm{~cm}^{2}$ ). Underlying liver diseases included hepatitis B $(29 / 44,66 \%)$, hepatitis C $(8 / 44,18 \%)$, and alcoholic liver disease $(5 / 44,11 \%)$ (Table 2$)$. Among the 44 participants, the best RECIST responses were a partial response in $6(14 \%)$ participants, stable disease in 18 (41\%) participants, progressive disease in $19(43 \%)$ participants, and a none-valuable response in $1(2 \%)$ 
participant. All participants had died by December 2018. The 3-month and 6-month PFS rates were $48 \%(21 / 44)$ and $11 \%(5 / 44)$, respectively. Median PFS and OS were 2.3 (range, $0.8-16.8$ ) and 8.0 (range, 1-54) months, respectively.

Table 2. Clinical characteristics and MRI parameters of 44 participants.

\begin{tabular}{cc}
\hline Characteristic & Value \\
\hline Age (y) & $60.0 \pm 11.6$ \\
Sex (men/women) & $39 / 5$ \\
Size $\left(\mathrm{cm}^{2}\right)$ & $38.4 \pm 44.4$ \\
ECOG $(0 / 1)$ & $8 / 36$ \\
Child-Pugh score (5/6) & $23 / 21$ \\
HBsAg (+) & $29(66)$ \\
Anti-HCV (+) & $8(18)$ \\
Alcohol abuse ${ }^{\dagger}$ & $5(11)$ \\
Cirrhosis ${ }^{+\dagger}$ & $34(77)$ \\
Extrahepatic metastasis & $38(86)$ \\
Macroscopic vascular invasion & $24(55)$ \\
Serum AFP $>400$ ng/mL & $29(66)$ \\
\hline Surgery & \\
Ablation & Prior treatment \\
TACE & $8(18)$ \\
Sorafenib & $35(80)$ \\
\hline
\end{tabular}

Note: Unless otherwise indicated, data are mean \pm standard deviation, and data in parentheses are percentages $\mathrm{AFP}=$ alpha-fetoprotein; $\mathrm{TACE}=$ transarterial chemoembolization; $\mathrm{ECOG}=$ Eastern Cooperative Oncology Group; $\mathrm{HBsAg}=$ hepatitis B surface antigen; Anti-HCV = antihepatitis $\mathrm{C}$ antibody. ${ }^{\dagger}$ Alcohol abuse was defined as history of more than 3 drinks a day, documentation of alcoholism or alcohol abuse in a physician's progress notes, or a history of alcoholic hepatitis. ${ }^{+\dagger}$ Cirrhosis was graded histologically $(n=4)$ or clinically (combined laboratory data and imaging, $n=30$ ).

\subsection{Correlation of Tumor Size, DCE-MRI, and IVIM Parameters}

The data of MRI parameters are shown in Table 3. Tumor size showed moderate inverse correlations with $\mathrm{ADC}(\mathrm{rho}=-0.54, p<0.001)$ and $\mathrm{f}(\mathrm{rho}=-0.44, p=0.001)$. $\mathrm{D}^{*}$ showed weak correlations with $\mathrm{K}^{\text {trans }}(\mathrm{rho}=0.26, p=0.045)$ and $\mathrm{K}_{\mathrm{ep}}(\mathrm{rho}=0.26, p=0.043)$. $\mathrm{ADC}$ and $\mathrm{D}$ were moderately correlated with each other $(\mathrm{rho}=0.41, p=0.005)$.

Table 3. MRI parameters of 44 participants.

\begin{tabular}{cc}
\hline & MRI Parameters \\
\hline Peak (\%) & $27.5 \pm 10.5$ \\
Slope $(1 / \mathrm{s})$ & $19.5 \pm 7.8$ \\
AUC $(/ 10 \mathrm{~s})$ & $2635 \pm 1101$ \\
$\mathrm{~K}^{\text {trans }}\left(\mathrm{min}^{-1} / 1000\right)$ & $152 \pm 146$ \\
$\mathrm{~K}_{\mathrm{ep}}\left(\mathrm{min}^{-1} / 1000\right)$ & $1252 \pm 1248$ \\
$\mathrm{~V}_{\mathrm{e}}(\%)$ & $10.7 \pm 6.2$ \\
$\mathrm{ADC}\left(10^{-3} \mathrm{~mm}^{2} / \mathrm{s}\right)$ & $1.4 \pm 0.3$ \\
$\mathrm{D}\left(10^{-3} \mathrm{~mm}^{2} / \mathrm{s}\right)$ & $1.1 \pm 0.3$ \\
$\mathrm{D}^{*}\left(10^{-3} \mathrm{~mm}^{2} / \mathrm{s}\right)$ & $65.1 \pm 38$ \\
$\mathrm{f}(\%)$ & $17.6 \pm 10.6$
\end{tabular}

Note: $\mathrm{AUC}$ = area under the curve; $\mathrm{K}^{\text {trans }}$ = forward volume transfer constant; $\mathrm{K}_{\mathrm{ep}}$ = reverse rate transfer constant $\mathrm{V}_{\mathrm{e}}=$ extravascular extracellular space volume per unit volume of tissue; $\mathrm{ADC}=$ apparent diffusion coefficient; $\mathrm{D}=$ pure diffusion coefficient; $\mathrm{D}^{*}=$ pseudodiffusion coefficient; $\mathrm{f}=$ perfusion fraction.

\subsection{Intraclass Correlation Coefficients of $M R$ Quantitative Parameters}

Intraclass correlation coefficients for interobserver variability were 1.00 (95\% CI: 1.00, 0.999 ) for peak, 0.999 (95\% CI: 0.998, 0.999) for slope, 0.999 (95\% CI: 0.998, 0.999) for AUC, 0.998 (95\% CI: 0.996, 0.999) for $\mathrm{K}^{\text {trans }}, 0.996$ (95\% CI: 0.993,0.998) for $\mathrm{K}_{\mathrm{ep}}, 0.992$ (95\% CI: 
$0.985,0.996)$ for $\mathrm{V}_{\mathrm{e}}, 0.935$ (95\% CI: $\left.0.883,0.964\right)$ for $\mathrm{ADC}, 0.927$ (95\% CI: 0.860, 0.961) for D, 0.943 (95\% CI: $0.799,0.977)$ for $\mathrm{D}^{*}$, and 0.975 (95\% CI: 0.944, 0.987) for $\mathrm{f}$.

3.4. Comparison of MRI Parameters Derived for Participants with Short ( $\leq 8$ Months) and Long (>8 Months) OS

Both ADC $(p=0.02)$ and $\mathrm{f}(p=0.02)$ were significantly higher in participants with a long OS than in those with a short OS. However, both parameters were not significant after Bonferroni correction was performed. DCE-MRI parameters did not significantly differ between participants in these two subgroups (Table 4).

Table 4. Comparison of MR parameters in participants with short ( $\leq 8$ months) and long ( $>8$ months) overall survival.

\begin{tabular}{cccc}
\hline Parameters & Short $(\boldsymbol{n}=\mathbf{2 3})$ & Long $(\boldsymbol{n}=\mathbf{2 1})$ & $p$ Value \\
\hline Peak $(\%)$ & $270 \pm 101$ & $280 \pm 112$ & 0.85 \\
Slope $(1 / \mathrm{s})$ & $18.8 \pm 7.2$ & $20.4 \pm 8.6$ & 0.45 \\
$\mathrm{AUC}(/ 10 \mathrm{~s})$ & $2660 \pm 1031$ & $2607 \pm 1199$ & 0.83 \\
$\mathrm{~K}^{\text {trans }}\left(\mathrm{min}^{-1} / 1000\right)$ & $106 \pm 75$ & $203 \pm 186$ & 0.08 \\
$\mathrm{~K}_{\mathrm{ep}}\left(\mathrm{min}^{-1} / 1000\right)$ & $943 \pm 647$ & $1591 \pm 1630$ & 0.10 \\
$\mathrm{~V}_{\mathrm{e}}(\%)$ & $9.6 \pm 5.4$ & $11.9 \pm 7$ & 0.36 \\
$\mathrm{ADC}\left(10^{-3} \mathrm{~mm}^{2} / \mathrm{s}\right)$ & $1.28 \pm 0.2$ & $1.54 \pm 0.4$ & $0.02^{*}$ \\
$\mathrm{D}\left(10^{-3} \mathrm{~mm}^{2} / \mathrm{s}\right)$ & $1.06 \pm 0.2$ & $1.14 \pm 0.4$ & 0.93 \\
$\mathrm{D}^{*}\left(10^{-3} \mathrm{~mm}^{2} / \mathrm{s}\right)$ & $65.6 \pm 38.8$ & $64.5 \pm 38$ & 0.87 \\
$\mathrm{f}(\%)$ & $14.6 \pm 9.1$ & $20.8 \pm 11.5$ & $0.02 *$ \\
\hline
\end{tabular}

Note: Data are mean \pm standard deviation. $A U C=$ area under the curve; $\mathrm{K}^{\text {trans }}=$ forward volume transfer constant; $\mathrm{K}_{\mathrm{ep}}=$ reverse rate transfer constant; $\mathrm{V}_{\mathrm{e}}=$ extravascular extracellular space volume per unit volume of tissue; $\mathrm{ADC}=$ apparent diffusion coefficient; $\mathrm{D}=$ pure diffusion coefficient; $\mathrm{D}^{*}=$ pseudodiffusion coefficient; $\mathrm{f}=$ perfusion fraction. ${ }^{*} p$ value indicates a significant difference. Employed statistical analysis was the MannWhitney test. Short and long OS were determined by a median OS period of 8.0 months.

\subsection{Correlation of MRI Parameters with PFS and OS}

Univariate analysis revealed that participants with a high slope $(p=0.024), \mathrm{K}_{\mathrm{ep}}$ $(p<0.0001)$, and ADC $(p=0.018)$ values had longer PFS than those with low values did (Table 5, Figure 5A-C). Large tumor size was a prognostic factor for poor OS $(p=0.006)$. Furthermore, participants with a high slope $(p=0.01)$, ADC $(p=0.015)$, and $\mathrm{f}(p=0.012)$ values had longer OS than those with low values (Table 5, Figure 5D-F). After incorporating significant variables, Cox multivariable analysis revealed that $\mathrm{K}_{\mathrm{ep}}$ (hazard ratio $=0.2 ; 95 \%$ confidence interval $=0.1,0.5 ; p<0.001$ ) and ADC (hazard ratio $=0.3 ; 95 \%$ confidence interval $=0.1,0.7 ; p=0.009$ ) remained independent predictors of PFS; slope (hazard ratio $=0.3 ; 95 \%$ confidence interval $=0.2,0.7 ; p=0.003$ ) and $\mathrm{ADC}$ (hazard ratio $=0.3 ; 95 \%$ confidence interval $=0.1,0.8 ; p=0.009$ ) remained independent predictors of OS. Moreover, $\mathrm{K}_{\mathrm{ep}}$ and slope were still significant after Bonferroni correction was performed.

Table 5. Univariate and multivariable Cox regression analysis for progression-free and overall survival.

\begin{tabular}{|c|c|c|c|c|c|c|c|c|c|}
\hline \multicolumn{2}{|c|}{ Parameters } & \multicolumn{4}{|c|}{ Progression-Free Survival (PFS) } & \multicolumn{4}{|c|}{ Overall Survival (OS) } \\
\hline & & \multicolumn{2}{|c|}{ Univariate } & \multicolumn{2}{|c|}{ Multivariable } & \multicolumn{2}{|c|}{ Univariate } & \multicolumn{2}{|c|}{ Multivariable } \\
\hline & Cutoff & $\begin{array}{c}\text { Hazard } \\
\text { Ratio }\end{array}$ & $p$ Value & $\begin{array}{c}\text { Hazard } \\
\text { Ratio }\end{array}$ & $p$ Value & $\begin{array}{c}\text { Hazard } \\
\text { Ratio }\end{array}$ & $p$ Value & $\begin{array}{c}\text { Hazard } \\
\text { Ratio }\end{array}$ & $p$ Value \\
\hline Age (y) & 60 & $1.0(0.5-1.8)$ & $>0.99$ & & & $1.0(0.6-1.9)$ & 0.89 & & \\
\hline sex & M vs. F & $0.5(0.2-1.3)$ & 0.18 & & & $1.0(0.4-2.4)$ & 0.98 & & \\
\hline Size $\left(\mathrm{cm}^{2}\right)$ & 17.5 & $0.9(0.5-1.7)$ & 0.82 & & & $0.4(0.2-0.8)$ & $0.006^{*}$ & & \\
\hline ECOG & 0 vs. 1 & $0.9(0.4-2.1)$ & 0.89 & & & $1.0(0.4-2.1)$ & 0.93 & & \\
\hline Child & 5 vs. 6 & $1.1(0.6-2.0)$ & 0.74 & & & $1.2(0.7-2.3)$ & 0.48 & & \\
\hline $\operatorname{AFP}(\mathrm{ng} / \mathrm{mL})$ & 400 & $0.9(0.5-1.7)$ & 0.78 & & & $0.8(0.4-1.5)$ & 0.49 & & \\
\hline
\end{tabular}


Table 5. Cont.

\begin{tabular}{|c|c|c|c|c|c|c|c|c|c|}
\hline \multicolumn{2}{|c|}{ Parameters } & \multicolumn{4}{|c|}{ Progression-Free Survival (PFS) } & \multicolumn{4}{|c|}{ Overall Survival (OS) } \\
\hline & & \multicolumn{2}{|c|}{ Univariate } & \multicolumn{2}{|c|}{ Multivariable } & \multicolumn{2}{|c|}{ Univariate } & \multicolumn{2}{|c|}{ Multivariable } \\
\hline & Cutoff & $\begin{array}{c}\text { Hazard } \\
\text { Ratio }\end{array}$ & $p$ Value & $\begin{array}{c}\text { Hazard } \\
\text { Ratio }\end{array}$ & $p$ Value & $\begin{array}{c}\text { Hazard } \\
\text { Ratio }\end{array}$ & $p$ Value & $\begin{array}{c}\text { Hazard } \\
\text { Ratio }\end{array}$ & $p$ Value \\
\hline Cirrhosis & $\begin{array}{l}\text { No vs. } \\
\text { yes }\end{array}$ & $0.7(0.4-1.5)$ & 0.39 & & & $0.9(0.4-1.8)$ & 0.71 & & \\
\hline $\begin{array}{l}\text { Macroscopic } \\
\text { vascular } \\
\text { invasion }\end{array}$ & $\begin{array}{l}\text { No vs. } \\
\text { yes }\end{array}$ & $0.99(0.5-1.8)$ & 0.98 & & & $1.8(1.0-3.4)$ & 0.06 & & \\
\hline \multirow[t]{2}{*}{$\begin{array}{c}\text { Extrahepatic } \\
\text { spread }\end{array}$} & $\begin{array}{l}\text { No vs. } \\
\text { yes }\end{array}$ & $1.1(0.4-2.8)$ & 0.89 & & & $1.0(0.4-2.7)$ & 0.97 & & \\
\hline & PFS/OS & & & & & & & & \\
\hline Peak (\%) & $32.5 / 16.8$ & $0.7(0.4-1.4)$ & 0.35 & & & $0.6(0.3-1.4)$ & 0.23 & & \\
\hline Slope $(1 / \mathrm{s})$ & $21.4 / 22.4$ & $0.5(0.3-0.9)$ & $0.024 *$ & $0.6(0.3-1.1)$ & 0.11 & $0.4(0.2-0.8)$ & $0.01 *$ & $0.3(0.2-0.7)$ & $0.003 *$ \\
\hline $\operatorname{AUC}(/ 10 \mathrm{~s})$ & $1115 / 3689$ & $0.5(0.2-1.3)$ & 0.15 & & & $1.7(0.8-3.7)$ & 0.19 & & \\
\hline $\begin{array}{c}\mathrm{K}^{\text {trans }} \\
\left(\mathrm{min}^{-1} / 1000\right)\end{array}$ & $51 / 21$ & $0.8(0.4-1.5)$ & 0.47 & & & $1.7(0.5-5.5)$ & 0.42 & & \\
\hline $\begin{array}{c}\mathrm{K}_{\mathrm{ep}} \\
\left(\mathrm{min}^{-1} / 1000\right)\end{array}$ & $370 / 1730$ & $0.2(0.1-0.5)$ & $<0.001$ * & $0.2(0.1-0.5)$ & $<0.001^{*}$ & $0.7(0.3-1.5)$ & 0.3 & & \\
\hline $\mathrm{V}_{\mathrm{e}}(\%)$ & $6.5 / 11.1$ & $0.7(0.4-1.4)$ & 0.34 & & & $1.6(0.9-3.0)$ & 0.11 & & \\
\hline $\begin{array}{c}\mathrm{ADC}\left(10^{-3}\right. \\
\left.\mathrm{mm}^{2} / \mathrm{s}\right)\end{array}$ & $0.943 / 1.138$ & $0.3(0.1-0.7)$ & $0.018^{*}$ & $0.3(0.1-0.7)$ & $0.009 *$ & $0.5(0.2-0.9)$ & $0.015^{*}$ & $0.3(0.1-0.8)$ & 0.009 * \\
\hline $\begin{array}{l}\mathrm{D}\left(10^{-3}\right. \\
\left.\mathrm{mm}^{2} / \mathrm{s}\right)\end{array}$ & $1.183 / 1.173$ & $0.6(0.2-1.3)$ & 0.2 & & & $0.6(0.3-1.3)$ & 0.18 & & \\
\hline $\begin{array}{l}\mathrm{D}^{*}\left(10^{-3}\right. \\
\left.\mathrm{mm}^{2} / \mathrm{s}\right)\end{array}$ & $10.9 / 10.9$ & $2.5(0.8-8.4)$ & 0.1 & & & $3.1(0.9-10.3)$ & 0.051 & & \\
\hline $\mathrm{f}(\%)$ & $28 / 23.4$ & $0.6(0.2-1.4)$ & 0.17 & & & $0.4(0.2-0.8)$ & $0.012 *$ & $0.6(0.3-1.1)$ & 0.1 \\
\hline
\end{tabular}

Note-Data in parentheses are $95 \%$ confidence intervals. AUC = area under the curve; $\mathrm{K}^{\text {trans }}=$ forward volume transfer constant; $\mathrm{K}_{\mathrm{ep}}=$ reverse rate transfer constant; $\mathrm{V}_{\mathrm{e}}=$ extravascular extracellular space volume per unit volume of tissue; $\mathrm{ADC}=$ apparent diffusion coefficient; $\mathrm{D}=$ pure diffusion coefficient; $\mathrm{D}^{*}=$ pseudodiffusion coefficient; $\mathrm{f}=$ perfusion fraction. ${ }^{*} p$ value indicates significant difference as determined by Cox regression analysis.
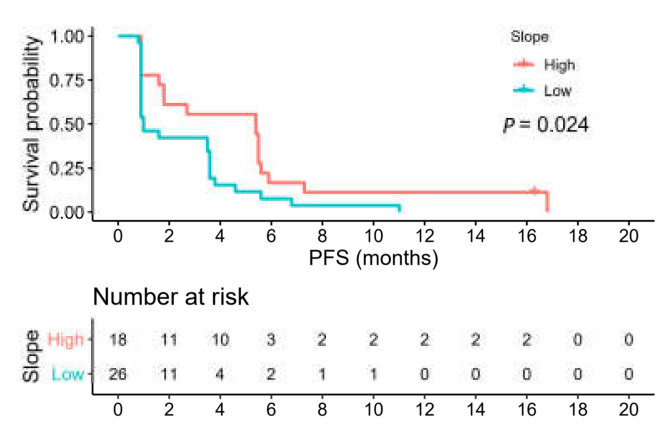

(A)

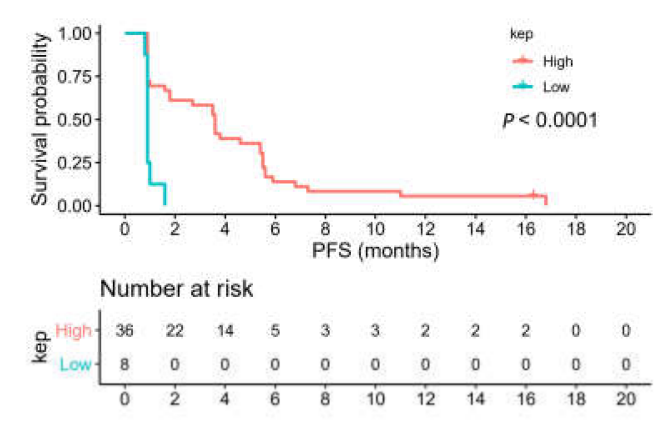

(B)

Figure 5. Cont. 

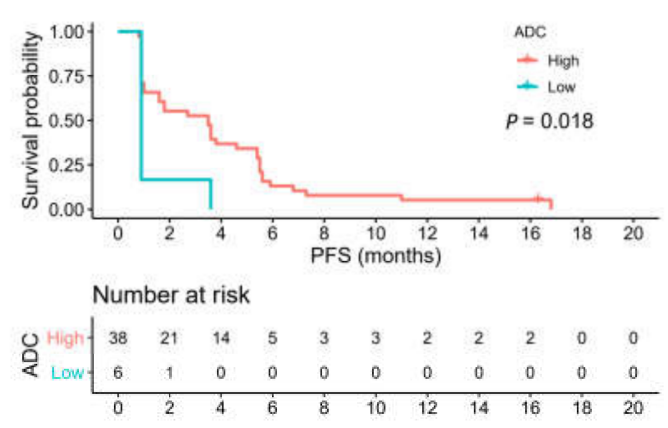

(C)

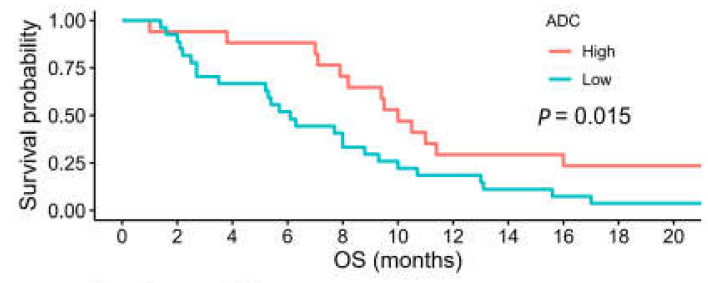

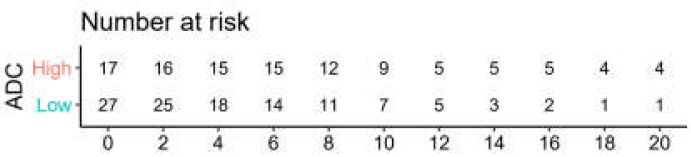

(E)
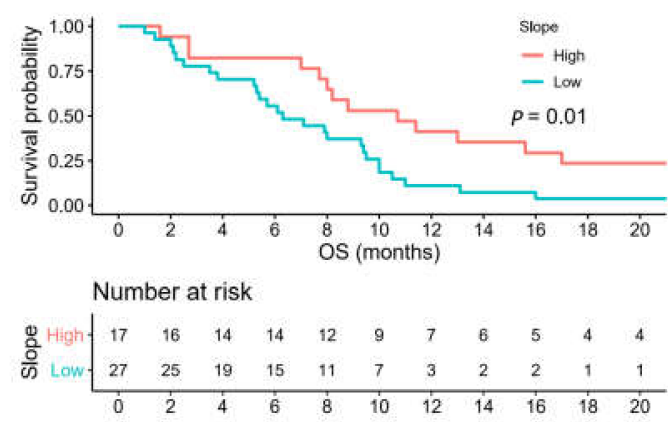

(D)

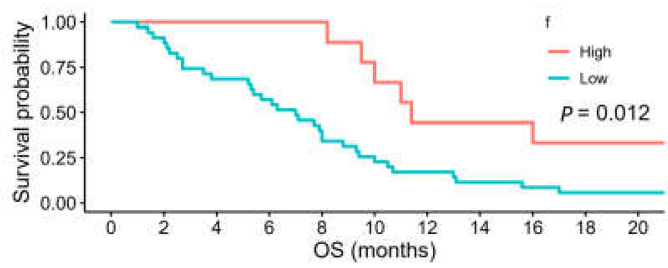

Number at risk

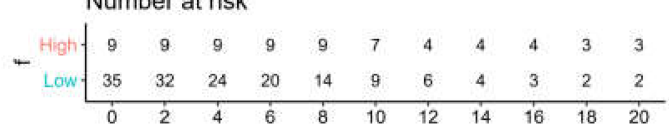

(F)

Figure 5. Kaplan-Meier curves indicating that participants with high pretreatment (A) slope, (B) $K_{\mathrm{ep}}$, and (C) ADC values had longer PFS than those with low values did. High pretreatment (D) slope, (E) ADC, and (F) f values had a longer OS than those with low values did. Cutoffs for MRI biomarkers were determined by using maximally selected rank statistics (maxstat package) in R statistical software.

\section{Discussion}

Our study demonstrated that, in participants with advanced HCC who received lenalidomide as second-line therapy, high baseline slope, $\mathrm{K}_{\mathrm{ep}}$, and ADC values were associated with better PFS, while small tumor size, high baseline slope, ADC, and $\mathrm{f}$ values were associated with better OS. Moreover, $\mathrm{K}_{\mathrm{ep}}$ and $\mathrm{ADC}$ remained independent predictors of PFS, and slope and ADC remained independent predictors of OS in multivariable analysis, after adjusting clinical factors and tumor size.

DCE-MRI provides both semiquantitative (peak, slope, and AUC) and quantitative $\left(\mathrm{K}^{\text {trans }}, \mathrm{K}_{\mathrm{ep}}, \mathrm{V}_{\mathrm{e}}\right.$ ) parameters for assessing tumor angiogenesis [8]. Previous studies reported that high baseline peak and an early decrease in peak within 1 week were associated with more favorable OS in participants with HCC receiving antiangiogenic therapy $[9,24]$. In contrast, our study demonstrated that a high baseline slope value was associated with more favorable PFS and OS. Another study also revealed that high baseline peak and slope values derived for a tumor before radiotherapy were strongly correlated with a more favorable RECIST-based response rate [25]. Slope is closely related to tumor blood flow, whereas peak represents a complex process involving perfusion, blood flow, and vascular permeability within tumors [24]. These semiquantitative parameters have potential for predicting participants' prognosis and have the advantage of easy calculation in daily practice; nevertheless, they may not be reproducible for different MRI machines or contrast injection flow rates. Therefore, standard and consistent MRI protocols are necessary for evaluating a treatment response or comparing results in multicenter clinical trials. 
DWI enables the quantitative assessment of tumor cellularity according to ADC values without the use of contrast agents; therefore, it is particularly useful in participants with severe renal dysfunction who are at risk of nephrogenic systemic fibrosis [12]. A retrospective study involving 58 patients with HCC who underwent drug-eluting embolic chemoembolization or radioembolization found that a lower ADC value was associated with a poorer PFS $(p=0.02)$ [13]. Another study used DCE-MRI and DWI in 20 patients with locally advanced HCC who had no treatment before concurrent chemoradiotherapy; the study revealed that patients with higher ADC values had significantly longer PFS than those with lower ADC values [26]. These findings are consistent with our results that a high pretreatment ADC value, representing low tumor cellularity or high tumor necrosis, was associated with more favorable survival outcomes.

Regarding IVIM DWI, $\mathrm{f}$ is believed to represent the fractional blood volume of microcirculation. $\mathrm{f}$ is also influenced by other bulk flow phenomena, including glandular secretion and the blood flow pattern [27]. Previous studies demonstrated a significant correlation between $\mathrm{f}$ and the percentage of arterial enhancement [28] or enhancement ratios [20] in HCC. Therefore, f may represent the hypervascular portion of a tumor. Furthermore, f was significantly correlated with treatment response to sorafenib in HCC, and increased $f$ after treatment suggested longer OS [29]. Although slope and $f$ are both related to microcirculations in a tumor, we did not find a significant correlation between these two parameters. Therefore, slope and $\mathrm{f}$ may reflect different pathophysiologies of the tumor microenvironment in advanced HCCs.

We identified a weak positive correlation of $\mathrm{D}^{*}$ with $\mathrm{K}^{\text {trans }}$ (rho $=0.26, p=0.045$ ) and $\mathrm{K}_{\mathrm{ep}}(\mathrm{rho}=0.26, p=0.043) . \mathrm{D}^{*}$ represents the length and velocity of the capillary network in a tumor [27], whereas $\mathrm{K}^{\text {trans }}$ and $\mathrm{K}_{\mathrm{ep}}$ both represent vascular permeability. $\mathrm{K}^{\text {trans }}$ and $\mathrm{K}_{\mathrm{ep}}$ were reported to predict treatment response or survival outcome in patients with HCC receiving antiangiogenic therapy $[11,30]$. In this study, we also found that high baseline $\mathrm{K}_{\mathrm{ep}}$ was associated with better PFS, but not OS.

Although ADC and D were moderately correlated with each other (rho $=0.41$, $p=0.005$ ), we did not find the correlation of D with clinical outcomes. ADC incorporates the information of tumor perfusion (low $b$ values) and cellularity (high $b$ values). It seems that tumor perfusion, rather than cellularity, was more likely to predict survival outcomes in our study population, probably due to the hypervascular characteristics of $\mathrm{HCC}$ and antiangiogenic effect of lenalidomide. Similarly, slope and $\mathrm{K}_{\mathrm{ep}}$ were also related to tumor perfusion, and both were independent predictors for survival outcomes.

Our study has several limitations. First, our sample size was small, and all patients were enrolled from a single institute. Second, all MRI parameters were measured on a single slice containing the largest tumor cross-sectional area instead of the whole tumor. Third, the acquisition time for IVIM was relatively long because multiple $b$ values were obtained [31]. A previous study found that D and $\mathrm{f}$ calculated by the simplified IVIM model from three $b$ values provided more discriminatory power between liver lesions than ADC determined from two $b$ values did [32]. Fourth, the use of least-squares fitting can introduce biases for the estimation of $f$ and $D^{*}$, and this can be improved using recent Bayesian approaches because they allow for the use of prior information to regularize the fitting and to introduce a spatial dependency between voxels [33,34]. Future study is necessary to compare the goodness-of-fit between the biexponential IVIM model and recent Bayesian approaches. Lastly, because DCE-MRI was performed on a coronal view, and T1WI/DWI/IVIM were performed on an axial view, we did not coregister these images. In a future study, we aim to produce these images on an axial view for better visualization.

In conclusion, both pretreatment DCE-MRI and IVIM DWI biomarkers, especially slope and ADC, may predict survival outcomes in participants receiving lenalidomide as second-line therapy. These biomarkers provide information that transcends mere morphology and have potential for use in pretreatment selection of participants who are likely to benefit from second-line targeted therapy. 
Author Contributions: Conceptualisation, B.-B.C., Y.-Y.S., Z.-Z.L., C.-H.H., A.-L.C.; C.H., P.-C.L. and T.T.-F.S.; methodology, B.-B.C., Y.-Y.S., Z.-Z.L., C.-H.H., A.-L.C., C.H., P.-C.L. and T.T.-F.S.; software, B.-B.C. and T.T.-F.S.; validation, B.-B.C. and T.T.-F.S.; formal analysis, B.-B.C. and Y.-Y.S.; investigation, B.-B.C., Y.-Y.S., Z.-Z.L., C.-H.H., A.-L.C.; C.H., P.-C.L. and T.T.-F.S.; resources, A.-L.C. and T.T.-F.S.; data curation, B.-B.C., Y.-Y.S., Z.-Z.L., C.-H.H., A.-L.C.; and C.H.; writing-original draft preparation, B.-B.C. and Y.-Y.S.; writing-Review and Editing, B.-B.C., Y.-Y.S., Z.-Z.L., C.-H.H., A.-L.C.; C.H., P.C.L. and T.T.-F.S.; visualisation, B.-B.C.; supervision, A.-L.C., C.H. and T.T.-F.S.; project administration, Y.-Y.S., A.-L.C., C.H. and T.T.-F.S.; funding acquisition, B.-B.C., Y.-Y.S., A.-L.C., C.H. and T.T.-F.S. All authors have read and agreed to the published version of the manuscript.

Funding: This research was funded by National Taiwan University Hospital: NTUH 101-001863, NTUH 104-S2723, NTUH 105-S3096, Ministry of Science and Technology, Taiwan: NSC 100-2314-B002-053, NSC 102-2321-B-002 -008, MOST 103-2321-B-002 -084, MOST 104-2321-B- 002-030 and The APC was funded by Ministry of Science and Technology.

Institutional Review Board Statement: The studies were conducted according to the guidelines of the Declaration of Helsinki. This study has been reviewed and approved by the Institutional Research Ethics Committee, National Taiwan University Hospital (protocol code: NTUH-REC No. 201105063MB, date of approval: 2 August 2011).

Informed Consent Statement: All patients signed an informed consent form.

Data Availability Statement: The datasets generated during and/or analysed during the current study are available from the corresponding author upon reasonable request.

Conflicts of Interest: The authors have declared that no competing interests exist.

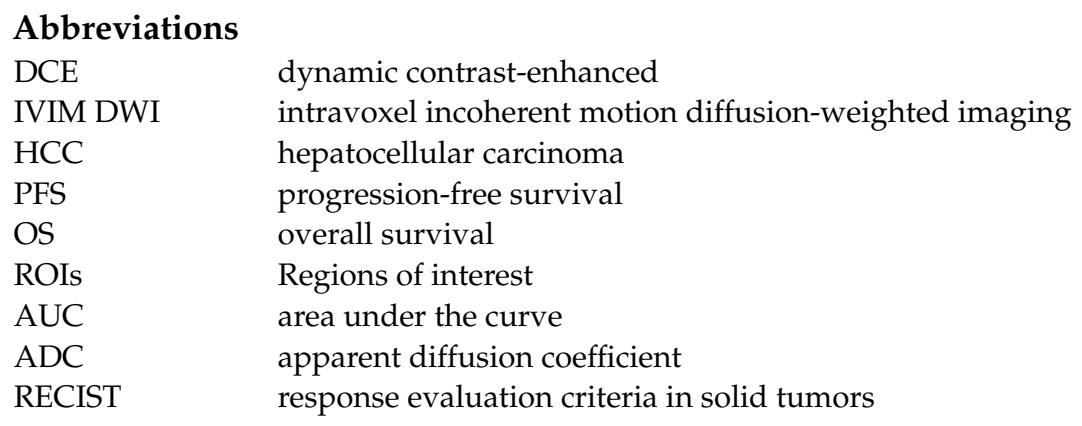

\section{References}

1. Bruix, J.; Sherman, M. Management of hepatocellular carcinoma: An update. Hepatology 2011, 53, 1020-1022. [CrossRef]

2. Forner, A.; Reig, M.; Bruix, J. Hepatocellular carcinoma. Lancet 2018, 391, 1301-1314. [CrossRef]

3. Cheng, A.L.; Kang, Y.K.; Chen, Z.; Tsao, C.J.; Qin, S.; Kim, J.S.; Luo, R.; Feng, J.; Ye, S.; Yang, T.S.; et al. Efficacy and safety of sorafenib in patients in the Asia-Pacific region with advanced hepatocellular carcinoma: A phase III randomised, double-blind, placebo-controlled trial. Lancet Oncol. 2009, 10, 25-34. [CrossRef]

4. Llovet, J.M.; Ricci, S.; Mazzaferro, V.; Hilgard, P.; Gane, E.; Blanc, J.F.; de Oliveira, A.C.; Santoro, A.; Raoul, J.L.; Forner, A.; et al. Sorafenib in advanced hepatocellular carcinoma. N. Engl. J. Med. 2008, 359, 378-390. [CrossRef] [PubMed]

5. Yarchoan, M.; Agarwal, P.; Villanueva, A.; Rao, S.; Dawson, L.A.; Llovet, J.M.; Finn, R.S.; Groopman, J.D.; El-Serag, H.B.; Monga, S.P.; et al. Recent Developments and Therapeutic Strategies against Hepatocellular Carcinoma. Cancer Res. 2019, 79, 4326-4330. [CrossRef]

6. Safran, H.; Charpentier, K.P.; Kaubisch, A.; Mantripragada, K.; Dubel, G.; Perez, K.; Faricy-Anderson, K.; Miner, T.; Eng, Y.; Victor, J.; et al. Lenalidomide for second-line treatment of advanced hepatocellular cancer: A Brown University oncology group phase II study. Am. J. Clin. Oncol. 2015, 38, 1-4. [CrossRef]

7. Shao, Y.Y.; Chen, B.B.; Ou, D.L.; Lin, Z.Z.; Hsu, C.H.; Wang, M.J.; Cheng, A.L.; Hsu, C. Lenalidomide as second-line therapy for advanced hepatocellular carcinoma: Exploration of biomarkers for treatment efficacy. Aliment. Pharmacol. Ther. 2017, 46, 722-730. [CrossRef]

8. Chen, B.B.; Shih, T.T. DCE-MRI in hepatocellular carcinoma-clinical and therapeutic image biomarker. World J. Gastroenterol. 2014, 20,3125-3134. [CrossRef]

9. Chen, B.B.; Hsu, C.Y.; Yu, C.W.; Liang, P.C.; Hsu, C.; Hsu, C.H.; Cheng, A.L.; Shih, T.T. Early perfusion changes within 1 week of systemic treatment measured by dynamic contrast-enhanced MRI may predict survival in patients with advanced hepatocellular carcinoma. Eur. Radiol. 2017, 27, 3069-3079. [CrossRef] [PubMed] 
10. Hsu, C.; Yang, T.S.; Huo, T.I.; Hsieh, R.K.; Yu, C.W.; Hwang, W.S.; Hsieh, T.Y.; Huang, W.T.; Chao, Y.; Meng, R.; et al. Vandetanib in patients with inoperable hepatocellular carcinoma: A phase II, randomized, double-blind, placebo-controlled study. J. Hepatol. 2012, 56, 1097-1103. [CrossRef] [PubMed]

11. Hsu, C.Y.; Shen, Y.C.; Yu, C.W.; Hsu, C.; Hu, F.C.; Hsu, C.H.; Chen, B.B.; Wei, S.Y.; Cheng, A.L.; Shih, T.T. Dynamic contrastenhanced magnetic resonance imaging biomarkers predict survival and response in hepatocellular carcinoma patients treated with sorafenib and metronomic tegafur/uracil. J. Hepatol. 2011, 55, 858-865. [CrossRef]

12. Taouli, B.; Koh, D.M. Diffusion-weighted MR imaging of the liver. Radiology 2010, 254, 47-66. [CrossRef]

13. Niekamp, A.; Abdel-Wahab, R.; Kuban, J.; Odisio, B.C.; Mahvash, A.; Hassan, M.M.; Qayyum, A.; Kaseb, A.; Sheth, R.A. Baseline Apparent Diffusion Coefficient as a Predictor of Response to Liver-Directed Therapies in Hepatocellular Carcinoma. J. Clin. Med. 2018, 7, 83. [CrossRef] [PubMed]

14. Zhu, S.C.; Liu, Y.H.; Wei, Y.; Li, L.L.; Dou, S.W.; Sun, T.Y.; Shi, D.P. Intravoxel incoherent motion diffusion-weighted magnetic resonance imaging for predicting histological grade of hepatocellular carcinoma: Comparison with conventional diffusionweighted imaging. World J. Gastroenterol. 2018, 24, 929-940. [CrossRef] [PubMed]

15. Shan, Q.; Chen, J.; Zhang, T.; Yan, R.; Wu, J.; Shu, Y.; Kang, Z.; He, B.; Zhang, Z.; Wang, J. Evaluating histologic differentiation of hepatitis B virus-related hepatocellular carcinoma using intravoxel incoherent motion and AFP levels alone and in combination. Abdom. Radiol. 2017, 42, 2079-2088. [CrossRef]

16. Wu, L.F.; Rao, S.X.; Xu, P.J.; Yang, L.; Chen, C.Z.; Liu, H.; Huang, J.F.; Fu, C.X.; Halim, A.; Zeng, M.S. Pre-TACE kurtosis of ADCtotal derived from histogram analysis for diffusion-weighted imaging is the best independent predictor of prognosis in hepatocellular carcinoma. Eur. Radiol. 2019, 29, 213-223. [CrossRef] [PubMed]

17. Wu, L.; Xu, P.; Rao, S.; Yang, L.; Chen, C.; Liu, H.; Fu, C.; Zeng, M. ADCtotal ratio and D ratio derived from intravoxel incoherent motion early after TACE are independent predictors for survival in hepatocellular carcinoma. J. Magn. Reson. Imaging 2017, 46, 820-830. [CrossRef]

18. Eisenhauer, E.A.; Therasse, P.; Bogaerts, J.; Schwartz, L.H.; Sargent, D.; Ford, R.; Dancey, J.; Arbuck, S.; Gwyther, S.; Mooney, M.; et al. New response evaluation criteria in solid tumours: Revised RECIST guideline (version 1.1). Eur. J. Cancer 2009, 45, $228-247$. [CrossRef] [PubMed]

19. Luciani, A.; Vignaud, A.; Cavet, M.; Nhieu, J.T.; Mallat, A.; Ruel, L.; Laurent, A.; Deux, J.F.; Brugieres, P.; Rahmouni, A. Liver cirrhosis: Intravoxel incoherent motion MR imaging-Pilot study. Radiology 2008, 249, 891-899. [CrossRef]

20. Kakite, S.; Dyvorne, H.A.; Lee, K.M.; Jajamovich, G.H.; Knight-Greenfield, A.; Taouli, B. Hepatocellular carcinoma: IVIM diffusion quantification for prediction of tumor necrosis compared to enhancement ratios. Eur. J. Radiol. Open 2016, 3, 1-7. [CrossRef]

21. Zhu, L.; Wang, H.; Zhu, L.; Meng, J.; Xu, Y.; Liu, B.; Chen, W.; He, J.; Zhou, Z.; Yang, X. Predictive and prognostic value of intravoxel incoherent motion (IVIM) MR imaging in patients with advanced cervical cancers undergoing concurrent chemoradiotherapy. Sci. Rep. 2017, 7, 11635. [CrossRef]

22. Le Bihan, D.; Breton, E.; Lallemand, D.; Aubin, M.L.; Vignaud, J.; Laval-Jeantet, M. Separation of diffusion and perfusion in intravoxel incoherent motion MR imaging. Radiology 1988, 168, 497-505. [CrossRef]

23. Rosset, A.; Spadola, L.; Ratib, O. OsiriX: An open-source software for navigating in multidimensional DICOM images. J. Digit. Imaging 2004, 17, 205-216. [CrossRef] [PubMed]

24. Chen, B.B.; Hsu, C.Y.; Yu, C.W.; Liang, P.C.; Hsu, C.; Hsu, C.H.; Cheng, A.L.; Shih, T.T. Dynamic Contrast-enhanced MR Imaging of Advanced Hepatocellular Carcinoma: Comparison with the Liver Parenchyma and Correlation with the Survival of Patients Receiving Systemic Therapy. Radiology 2016, 281, 454-464. [CrossRef]

25. Liang, P.C.; Ch'ang, H.J.; Hsu, C.; Chen, L.T.; Shih, T.T.; Liu, T.W. Perfusion parameters of dynamic contrast-enhanced magnetic resonance imaging predict outcomes of hepatocellular carcinoma receiving radiotherapy with or without thalidomide. Hepatol. Int. 2015, 9, 258-268. [CrossRef]

26. Kim, K.A.; Park, M.S.; Ji, H.J.; Park, J.Y.; Han, K.H.; Kim, M.J.; Kim, K.W. Diffusion and perfusion MRI prediction of progressionfree survival in patients with hepatocellular carcinoma treated with concurrent chemoradiotherapy. J. Magn. Reson. Imaging 2014, 39, 286-292. [CrossRef] [PubMed]

27. Le Bihan, D. Intravoxel incoherent motion perfusion MR imaging: A wake-up call. Radiology 2008, 249, 748-752. [CrossRef]

28. Woo, S.; Lee, J.M.; Yoon, J.H.; Joo, I.; Han, J.K.; Choi, B.I. Intravoxel incoherent motion diffusion-weighted MR imaging of hepatocellular carcinoma: Correlation with enhancement degree and histologic grade. Radiology 2014, 270, 758-767. [CrossRef]

29. Lewin, M.; Fartoux, L.; Vignaud, A.; Arrive, L.; Menu, Y.; Rosmorduc, O. The diffusion-weighted imaging perfusion fraction $\mathrm{f}$ is a potential marker of sorafenib treatment in advanced hepatocellular carcinoma: A pilot study. Eur. Radiol. 2011, 21, 281-290. [CrossRef]

30. Sahani, D.V.; Jiang, T.; Hayano, K.; Duda, D.G.; Catalano, O.A.; Ancukiewicz, M.; Jain, R.K.; Zhu, A.X. Magnetic resonance imaging biomarkers in hepatocellular carcinoma: Association with response and circulating biomarkers after sunitinib therapy. $J$. Hematol. Oncol. 2013, 6, 51. [CrossRef]

31. Wei, Y.; Huang, Z.; Tang, H.; Deng, L.; Yuan, Y.; Li, J.; Wu, D.; Wei, X.; Song, B. IVIM improves preoperative assessment of microvascular invasion in HCC. Eur. Radiol. 2019, 29, 5403-5414. [CrossRef] [PubMed]

32. Murtz, P.; Sprinkart, A.M.; Reick, M.; Pieper, C.C.; Schievelkamp, A.H.; Konig, R.; Schild, H.H.; Willinek, W.A.; Kukuk, G.M. Accurate IVIM model-based liver lesion characterisation can be achieved with only three b-value DWI. Eur. Radiol. 2018, 28, 4418-4428. [CrossRef] [PubMed] 
33. Gustafsson, O.; Montelius, M.; Starck, G.; Ljungberg, M. Impact of prior distributions and central tendency measures on Bayesian intravoxel incoherent motion model fitting. Magn. Reson. Med. 2018, 79, 1674-1683. [CrossRef] [PubMed]

34. Lanzarone, E.; Mastropietro, A.; Scalco, E.; Vidiri, A.; Rizzo, G. A novel bayesian approach with conditional autoregressive specification for intravoxel incoherent motion diffusion-weighted MRI. NMR Biomed. 2020, 33, e4201. [CrossRef] 